Conclusion: In this small study, seropositivity and disease activity were higher in responders. Baseline immunohistochemical staining was not a good discriminator of treatment respons

Disclosure of Interests: Kieran Murray Grant/research support from: Bresnihan Molloy and Newman fellowships, Candice Low: None declared, Francis Young: None declared, Monika Bienicka: None declared, Elmear Mylod: None declared, Ursula Fearon: None declared, Douglas Veale: None declared DOI: 10.1136/annrheumdis-2021-eular.2593

\section{POS0478 ASSOCIATION BETWEEN BODY SHAPES AND BODY SHAPE TRAJECTORIES, AND THE RISK OF RHEUMATOID ARTHRITIS IN THE FRENCH E3N COHORT}

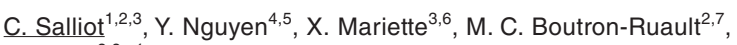
R. Seror ${ }^{3,8} .{ }^{1}$ Centre Hospitalier d'Orleans, Rheumatology, Orleans, France; ${ }^{2}$ Center for Research in Epidemiology and Population Health (CESP), Université Paris-Saclay, Institut pour la Santé et la Recherche Médicale (INSERM) U1018, Villejuif, France; ${ }^{3}$ Center of Immunology of Viral Infections and Auto-immune Diseases (IMVA), Université Paris-Saclay, Institut pour la Santé et la Recherche Médicale (INSERM) U1184, Le Kremlin Bicetre, France $;{ }^{4}$ Hôpital Beaujon, Université de Paris, Internal Medicine, Clichy, France; ${ }^{5}$ Center for Research in Epidemiology and Population Health (CESP), Paris-Saclay, Institut pour la Santé et la Recherche Médicale (INSERM) U1018, Villejuif, France; ${ }^{6}$ Assistance Publique-Hôpitaux de Paris (AP-HP), AP-HP. Université Paris-Saclay - Hôpital Bic être, Rheumatology, Kremlin Bicêtre, France; ${ }^{7}$ Institut Gustave Roussy, Universite Paris Saclay, Villejuif, France; ${ }^{8}$ Assistance Publique-Hôpitaux de Paris (AP-HP), AP-HP. Université Paris-Saclay - Hôpital Bicêtre, Rheumatology, Le Kremlin Bicetre, France

Background: Several cohort and case-control studies have suggested that overweight or obesity is associated with the risk of Rheumatoid Arthritis (RA). Associations were based on Body Mass Index (BMI), although this measurement does not reflect the fat distribution [1].

Objectives: To study the relationships between anthropometric measurements and the risk of RA in women involved in the E3N cohort.

Methods: E3N is an ongoing French prospective cohort that enrolled 98,995 women aged 40-65 years in 1990. Women completed mailed questionnaires every 2-3 years on lifestyle and health-related information. A total of 698 incident RA cases have been validated among 78,452 women [2]. Available anthropometric measurements include birth height and weight, height and weight (collected at baseline and regularly updated during follow-up), and age-related body shapes (BS). Women had to identify the silhouette among 8 BS that best described their BS at 8 years, puberty, 20-25 years, 30-35 years, and study baseline. BS trajectories (from 8 to $30-35$ years) were constructed using Nagin's approach to group-based trajectory modeling that identifies 6 different trajectories [3]. Hazard ratios (HRs) and 95\% confidence intervals (Cls) for the risk of incident RA were estimated using Cox proportional hazards regression models with age as the time scale. Models were first adjusted for known risk factors of RA (model 1), and then multi-adjusted (model 2).

Results: Taking lean BS as reference, medium BS at puberty was associated with an increased risk of $\mathrm{RA}[\mathrm{HR}=1.23(95 \% \mathrm{Cl} 1.0-1.5)]$, in the fully adjusted model (table 1), as was large BS at baseline $[\mathrm{HR}=1.32(95 \% \mathrm{Cl} 1.1-1.6)]$ (in model 1). Obesity $\left(B M l>30 \mathrm{~kg} / \mathrm{m}^{2}\right)$ was marginally associated with RA in model $1[\mathrm{HR}=1.30(95 \% \mathrm{Cl} 1.0-1.7)]$, but the association was no longer statistically

Table 1. Cox proportional-hazards analysis of anthropometric factors associated with RA

\begin{tabular}{|c|c|c|c|c|c|c|}
\hline & \multirow[t]{2}{*}{ RA } & \multirow[t]{2}{*}{ Non-cases } & \multicolumn{2}{|l|}{ MODEL 1} & \multicolumn{2}{|l|}{ MODEL 2} \\
\hline & & & HRs $(95 \% \mathrm{Cl})$ & ptrend & HRs $(95 \% \mathrm{Cl})$ & ptrend \\
\hline \multicolumn{7}{|c|}{ Baseline Body mass index $\left(\mathrm{kg} / \mathrm{m}^{2}\right)$} \\
\hline$<18$ & 10 & 1,797 & $0.75(0.4-1.4)$ & 0.0532 & $0.85(0.4-1.6)$ & 0.6165 \\
\hline [18-25[ & 462 & 50,330 & Ref & & Ref & \\
\hline$[25-30]$ & 171 & 19,716 & $1.10(0.9-1.3)$ & & $1.00(0.8-1.2)$ & \\
\hline$>30$ & 55 & 5,911 & $1.30(1.0-1.7)$ & & $1.13(0.8-1.6)$ & \\
\hline \multicolumn{7}{|c|}{ Body shape at puberty } \\
\hline Lean & 332 & 40,485 & Ref & 0.0937 & Ref & 0.1490 \\
\hline Medium & 179 & 17,903 & $1.24(1.0-1.5)$ & & $1.23(1.0-1.5)$ & \\
\hline Large & 147 & 15,984 & $1.14(0.9-1.4)$ & & $1.12(0.9-1.4)$ & \\
\hline \multicolumn{7}{|c|}{ Body shape at baseline } \\
\hline Lean & 359 & 44,718 & Ref & 0.0055 & Ref & 0.1743 \\
\hline Medium & 200 & 20,041 & $1.20(1.0-1.4)$ & & $1.16(0.9-1.4)$ & \\
\hline Large & 110 & 9,838 & $1.32(1.1-1.6)$ & & $1.20(0.9-1.6)$ & \\
\hline
\end{tabular}

ptrend: $\mathrm{p}$ for trend. Ref: reference. significant in the multi-adjusted model, taking normal BMI $\left[18-25 \mathrm{~kg} / \mathrm{m}^{2}\right.$ [as the reference.

Birth weight and height, BS (at 8, 20-25 and 30-35 years), and BS trajectories were not significantly associated with RA in any model.

Conclusion: In E3N cohort, medium body shape at puberty was associated with an increased risk of RA independently of the BMI and smoking exposure.

Model 1 adjusted for age, smoking (past/current/never), passive smoking during childhood and/or adulthood (ever/never), educational level (<high-school, up to 2 years of university, $\geq 3$ years of university).

Multi-adjusted model 2 included model $1+$ body mass index $(<18,18-25$ $25-30,>30 \mathrm{~kg} / \mathrm{m}^{2}$ ), body shape at puberty, body shape at baseline adjusted for age, baseline physical activity (in quartiles), age at menarche $(<13,13$ $15, \geq 15$ years), age at menopause ( $\leq 45,45-53, \geq 53$ years), age at the first pregnancy $(<22,22-27, \geq 27$ years), number of full-term pregnancies ( $\leq 1$ $2, \geq 3)$, and duration of premenopausal use of progestogen $(0,0-24,>24$ months).

\section{REFERENCES:}

[1] Crowson CS, Matteson EL, Davis JM 3rd, Gabriel SE. Contribution of obesity to the rise in incidence of rheumatoid arthritis. Arthritis Care Res (Hoboken) 2013:65:71-7.

[2] Nguyen Y, Salliot C, Gusto G, et al. Improving accuracy of self-reported diagnoses of rheumatoid arthritis in the French prospective E3N-EPIC cohort: a validation study. BMJ Open. 2019;9:e033536.

[3] Nagin D. Analyzing developmental trajectories: a semiparametric, group based approach. Psychol Methods 1999;4:139e57.

Disclosure of Interests: None declared

DOI: 10.1136/annrheumdis-2021-eular.2655

\section{\begin{tabular}{|l|l} 
POS0479 THE IMPACT OF FLARES ON PATIENT \\
\hline
\end{tabular} REPORTED OUTCOMES IN RHEUMATOID AND UNDIFFERENTIATED ARTHRITIS PATIENTS - A SUB- ANALYSIS OF THE IMPROVED STUDY}

J. M. Maassen ${ }^{1}$, S. A. Bergstra ${ }^{1}$, P. D. de Buck ${ }^{2}$, M. van Oosterhout ${ }^{3}$,

T. Huizinga', C. Allaart ${ }^{1}{ }^{1}$ Leiden University Medical Center (LUMC),

Rheumatology, Leiden, Netherlands; ${ }^{2}$ Haaglanden Medical Center,

Rheumatology, The Hague, Netherlands; ${ }^{3}$ Groene Hart Hospital, Rheumatology, Gouda, Netherlands

Background: In rheumatoid arthritis (RA) patients in low disease activity, flares are associated with symptom deterioration. Patients in clinical remission may flare but still have low disease activity. How does this affect patient reported outcomes?

Objectives: To evaluate the prevalence of disease flares in patients treated to target drug free remission, and to study the impact of disease flares on patient-reported outcomes (PROs) for flares with different impact on disease activity.

Methods: In the IMPROVED study 610 patients with rheumatoid arthritis (RA) or undifferentiated arthritis (UA) were treated to target drug free remission (DAS $<1.6$ ) for 5 years. As soon as DAS was $<1.6$, treatment was tapered to discontinuation. Patients with at least 8 months follow-up were selected. A flare was defined according to three definitions; A) DAS $\geq 1.6$ and $\geq 0.6$ increase from the previous visit regardless of the previous DAS; $\mathbf{B}$ ) minor flare from remission; a DAS $\geq 1.6$ with $<0.6$ increase and previous DAS $<1.6$; C) major flare from remission; a DAS $\geq 1.6$ with $\geq 0.6$ increase and previous DAS $<1.6$. Linear mixed models were used to compare functional ability, measured by the health assessment questionnaire $(\mathrm{HAQ})$, at visits where a flare occurred versus visits without a flare. Fisher's exact test were used to compare percentages with $\geq 0.22 \mathrm{HAQ}$ increases between groups with and without LDA at the moment of flare. A generalized linear mixed model was used to calculate the odds ratio for a deterioration of $\geq 20 \mathrm{~mm}$ in VAS of PROs global health $(\mathrm{GH})$, disease activity, pain and morning stiffness (from the preceding visit) during a flare.

Results: Of the 585 patients with sufficient follow-up, 75\% experienced a flare A, $26 \%$ a flare B, and $68 \%$ a flare C, at least once. Most flares were observed after $\mathrm{t}=8$ and $\mathrm{t}=12$ months. In $55 \%, 100 \%$, and $69 \%$ of visits with a flare $\mathrm{A}, \mathrm{B}$ or $\mathrm{C}$, the patients were still in LDA (DAS $=<2.4)$. In $55 \%$ of the visits where a flare was associated with a DAS increase $\geq 0.6$ (flare $A \& C$ ) there was also clinically relevant increase in $\mathrm{HAQ}$ of $\geq 0.22$. The mean difference in $\mathrm{HAQ}$ was 0.27 with flare $\mathbf{A}(\mathrm{p}<0.01), 0.03$ with flare $\mathbf{B}(\mathrm{p}=0.72)$ and 0.18 with flare $\mathbf{C}(\mathrm{p}<0.01)$. If was DAS $>2.4$ (LDA) at the moment of flare, HAQ increased $\geq 0.22$ in $68 \%$ of all flares $A$, and $77 \%$ of all flares $C$ (p-values $<0.01$, compared to flares where patients were still in LDA, DAS $=<2.4$ ). The odds ratios of a $>20 \mathrm{~mm}$ deterioration in VAS global health, VAS disease activity, VAS pain and VAS morning stiffness was significant $\geq 1$ for flares with $a \geq 0.6$ increase in DAS (flares $A$ and $C$ ), and $\leq 1$ for minor flares (B) (table 1). 